\title{
Evaluation of Gastric Diseases Using Segmentation RGB Interface in Video Endoscopy Images
}

\author{
Sandra Luz Mendoza-Trejoㄹ, Víctor Manuel Ruiz-Martinez ${ }^{1,2}$, José Ernesto \\ Domínguez-Herrera ${ }^{1,2}$
}

\author{
${ }^{1}$ Group of Polymer Composite Materials and Interphases, Carlos III University of Madrid, Av. Universidad 30, 28911 \\ Leganés, Spain \\ Email: 100367719@alumnos.uc3m.es \\ ${ }^{2}$ Department of IMI, Universidad Tecnológica del Centro de Veracruz, . Av.Universidad 350, 94910 Veracruz, México \\ Email: sandra.mendoza@utcv.edu.mx,victor.ruiz@utcv.edu.mx
}

\begin{abstract}
Video endoscopy is a prevention, diagnosis and prognosis technique usually used to detect gastric lesions due to its minimal invasion and low risk, however the critique of the medical personnel is the main actor to give a diagnosis, in this article, a novel algorithm of image processing using MATLAB software to separate video endoscopy in 6 tonalities implementing $R G B$ filters and their combinations has been evaluated by medical personnel to determinate if the colorimetry helps in the detection of gastric diseases such as polyps, varicose veins and ulcers, the medical staff indicates that the color red, pink (Without green) and yellow (without blue) help in the detection of varicose veins due to enhanced of natural color and reduction the interference of fluids in cavity, gastric ulcers are detected easily by yellow (without blue) and pink colors.
\end{abstract}

Keywords - RGB Segmentation, Video Endoscopy, Injury Identification.

\section{INTRODUCTION}

The human interest to explore the human body has transcended through time, since the eighteenth century the ability to observe and explore the cavities was reduced to the mouth, the oropharynx, the nostrils, the external auditory canal, the vagina and the rectum [1], later in 1806, Philipp Bozzini used a tube that he created, known as "Lichtleited" to study the urinary tract using the sun and a candle as a source of light to see further into the body's cavities [2]-[3], Antonín J. Desormeaux began the current endoscopy in 1965 with his study of the urinary tract and the bladder using an instrument called "endoscope"[4].

In 1868, Dr. Adolph Kussmaul, got to observe the inside of a stomach of a living human body for the first time. The test was performed with a swallow's swords that could swallow a long and straight metal tube [5]. In 1881, Johann Von
Mikulicz and his associates created the first rigid gastroscopy [6], but it was until 1932 that Dr. Rudolph Schindler invented a flexible gastroscope that allowed the examination of the interior of a stomach through numerous lenses located throughout the tube with a miniature electric lamp [7].

The use of the technology has allowed endoscopy studies were less invasive every time [8], with simpler interfaces [9] and with a more precise analysis using color models [10], while the advancement of technology the characteristics are improved, however these improvements are offset by the high costs of these technologies.

Nowadays, there are video endoscopy systems that use techniques based on edges and clusters to classify and show the images that are in focus allowing to improve the conditions to the specialist to take better decisions [11]. Those can show images in different shades of color by using chromatic filters, giving to the endoscopy specialist doctors precious information to the detection of lesions and diseases in the inner cavities [12] In the technological advances of the endoscopy video equipment, the FICE system is one (FUJI Intelligent Chromo Endoscopy), it uses spectral images to get images that remark the characteristics of the mucosa allowing to identify tissue lesions [13],the NBI (Narrow band imaging) is a video endoscopy system based in the modification of the light bandwidth emitted, using a system of filters that stretches the light wave length highlighting the vascular patterns [14]

Currently, video endoscopy is an indispensable tool for the detection and treatment of different diseases, however, when performing endoscopic examinations, there are some lesions that are not seen with the naked eye, so when observed in certain shades of color using Color filters, the image provides valuable information to medical specialists 
in endoscopy for the detection of lesions and diseases of the internal cavities with greater precision.

The current systems of endoscopy are excessively expensive, doubling or tripling in price of the traditional equipment, therefore, they are not easily acquired, due to the above, it is necessary to develop and evaluate a system for the spectral decomposition of video endoscopy, using filters in their color components to obtain images with tonalities that give information in the diagnosis of diseases.

\section{METHOD}

The MATLAB software was used in these studies, implementing filters in the space of color RGB in a sequence of endoscopy images.

The filter RGB works delimitating one or two components of color, in this investigation red, green, blue, without red (Blue-green), without green (pink), and without blue (yellow) colors were tested.

To use a specific configuration as red color, red color turn " 1 " while green and blue colors turn " 0 " as shows in table 1.

Table.1: RGB configuration

\begin{tabular}{|l|c|c|c|}
\hline \multirow{2}{*}{ RGB configuration } & \multicolumn{3}{|c|}{ Primary Colors } \\
\cline { 2 - 4 } & Red & Green & Blue \\
\hline Red & 1 & 0 & 0 \\
\hline Green & 0 & 1 & 0 \\
\hline Blue & 0 & 0 & 1 \\
\hline Without Red & 0 & 1 & 1 \\
\hline Without Green & 1 & 0 & 1 \\
\hline Without Blue & 1 & 1 & 0 \\
\hline
\end{tabular}

Figure 1 shows the block diagram of the image conversion, the original image is separated into its RGB components, then the filtering is done in the space domain, by the convolution, resulting in the spectral components $R^{\prime} G^{\prime} B^{\prime}$ in non-true color. Finally, the specters are joined to generate the resulting image.

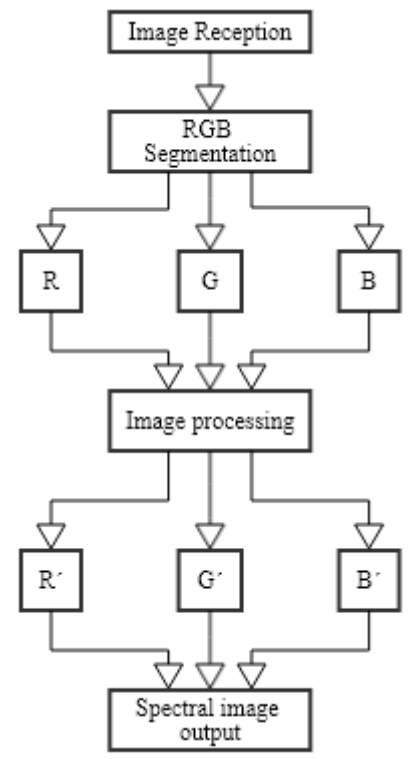

Fig.1: RGB segmentation block diagram
The endoscope-computer-user interface was programmed in MATLAB software to process video endoscopy; it shows the sequence of images red, green and blue, without red (blue-green), without green (pink), and without blue (yellow) colors following the code shows below.

Mov= aviread('prueba.avi');

$\mathrm{T}=\mathrm{size}(\mathrm{mov})$;

$\mathrm{f}=\mathrm{T}(2)$;

for $i=1: 5$

fram $=\operatorname{mov}(:, 1)$;

tim=size (fram. cdata) ;

mneg=uint $8(\operatorname{zeros}(\operatorname{tim}(1), \operatorname{tim}(2)))$;

for $j=1: f$

fram=mov $(:, j)$;

ImO=fram. cdata $(:,:,:)$;

$\operatorname{ImR}(:,:, 1)=$ fram. cdata $(:,:, 1)$;

$\operatorname{ImR}(:,:, 2)=$ zeros; $\operatorname{ImR}(:,:, 3)=$ zeros ;

Figure 2 MATLAB code sample

Injuries such as ulcers, polyps and varicose veins, were evaluated by video endoscopy, those were provided by specialized doctors, the images were processed and filtered in the RGB spectra, an information analysis was performed using an instrument that evaluated colorimetry, injury identification, coupling to equipment, and customer satisfaction.

\section{RESULTS}

\subsection{Man-Machine Interface}

The interface in figure 3 shows the system for selecting the desired RGB regime, allowing with one click to select the channel to filter, $67.5 \%$ of endoscopy specialists indicated that the interface is efficient and easy to use.

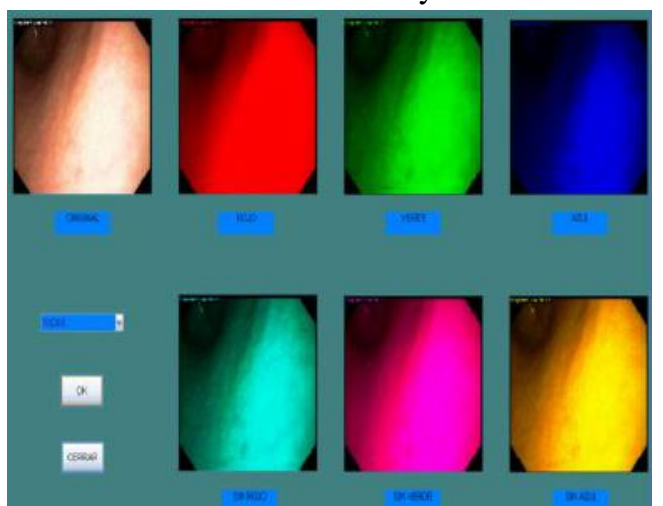

Fig.3: Graphic interface of the different components of the RGB system.

According to the injuries analyzed, it can be determined the injure to identify, because the $90 \%$ of specialists indicated by use of colorimetry allows better identification of this injury, followed by polyps and varicose veins with $58 \%$ and $53 \%$ respectively. 


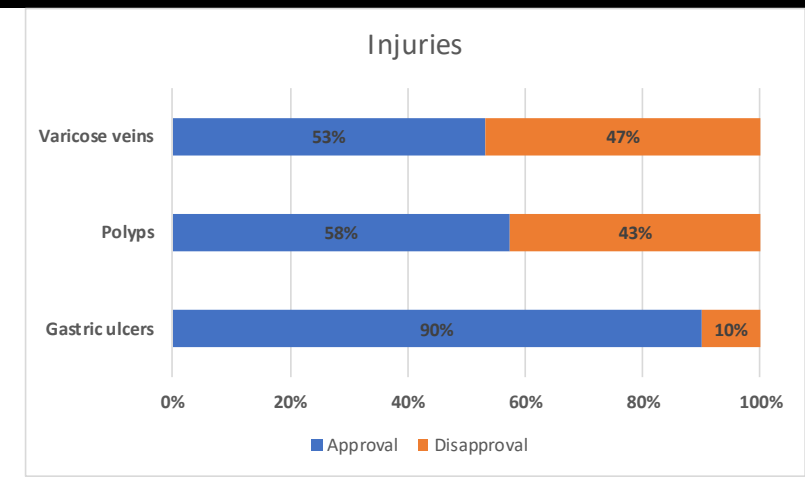

Fig.4: Approval for injury

The use of RGB colorimetry in the identification of varicose veins and polyps is effective, however, $42.5 \%$ of medical specialists say the color influences the analysis in these diseases, in the same way, only $20 \%$ medical specialist agree with colorimetry. It can affect on the analysis, emphasizing on green and blue colors.

\subsection{VIDEO ENDOSCOPY ANALYSIS.}

The use of different RGB tonalities were evaluated according to decomposition of original image in 6 combinations results in three conditions. This conditions are the original $\mathrm{R}, \mathrm{G}$ and $\mathrm{B}$ segmentation and combinations $\mathrm{R}$ G, G-B and R-B

\subsubsection{Varicose veins}

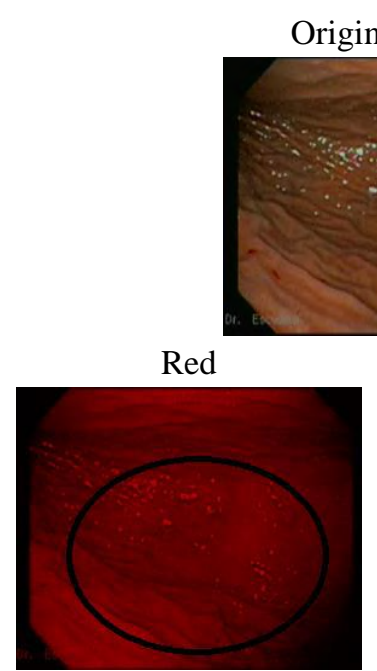

Blue

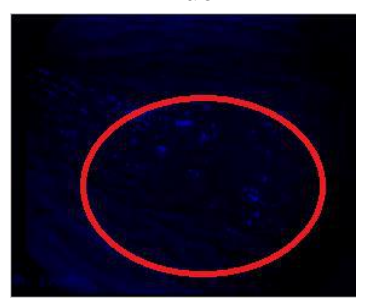

Green

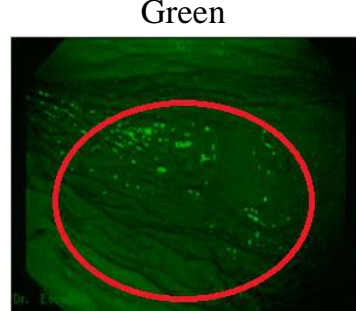

Yellow

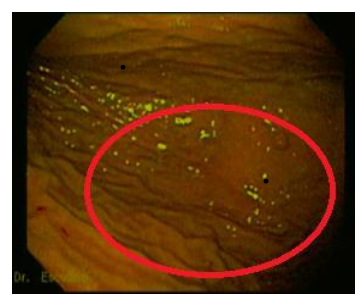

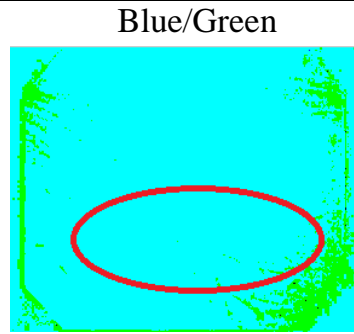

Pink

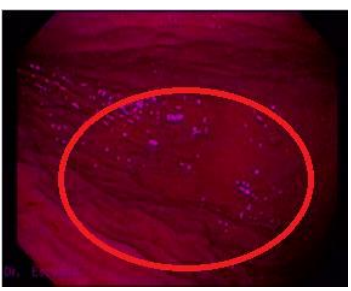

Fig.5: RGB Decomposition of Varicose veins

The detection of varicose veins is efficient using colors such as red, pink and yellow, because the tortuous or dilated vein is highlighted. The $50 \%$ of the specialists opted for the red tint to detect this lesion, followed by pink (without green) with $33 \%$ and color yellow (without blue) with $17 \%$.

The shades Green, blue green and blue, (without red) do not highlight the varicose vein in the gastric cavity, because the blue and green color show a colorimetric saturation, while the green color does not show evidence of the lesion.

\subsubsection{Gastric ulcer}

The suffering lesion by medical specialists to be detected is the ulcer because the $90 \%$ agrees that it is quick and easy to detect with the decomposition of colors, although, they explain to use yellow and pink (without green) colors are the most useful colors for this detection with $67 \%$ and $33 \%$ of effectiveness respectively.

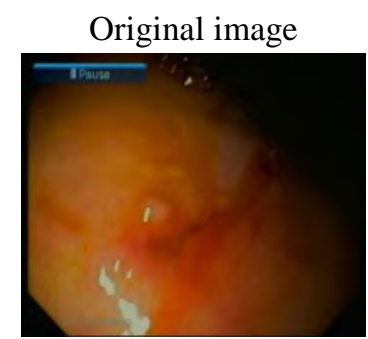

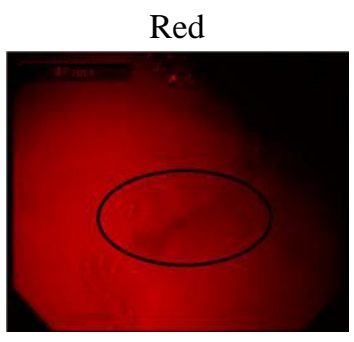

Blue

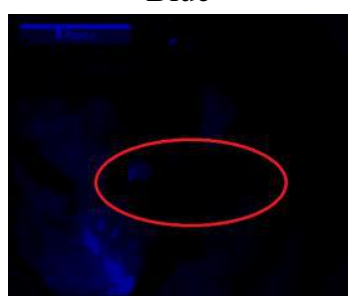

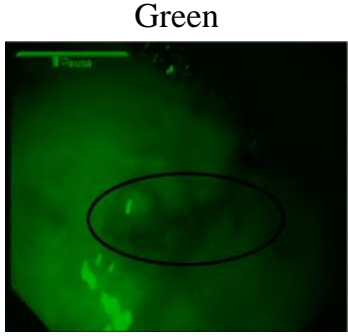

Yellow

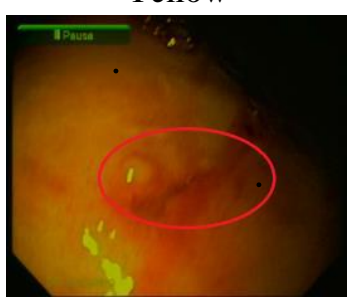



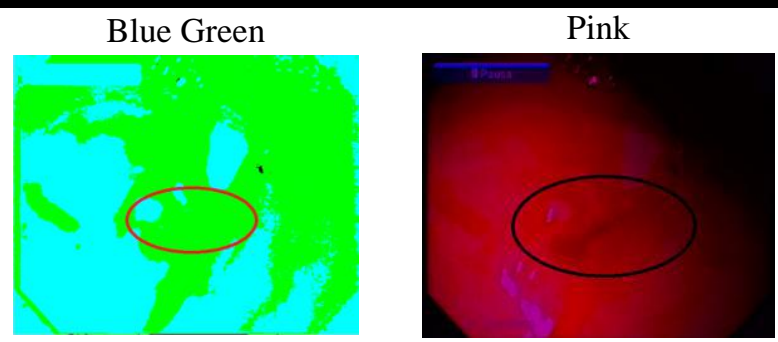

Fig.6: RGB Decomposition of Gastric ulcer

The primary colors and blue-green composition (without red) was descanted due the images saturated.

The medical specialists explain about filtration with yellow and pink colors (without green), allow to find small lesions in the upper small intestine, which early Zollinger Ellison syndrome.

The first paragraph under each heading or subheading should be flush left, and subsequent paragraphs should have a five-space indentation. A colon is inserted before an equation is presented, but there is no punctuation following the equation. All equations are numbered and referred to in the text solely by a number enclosed in a round bracket (i.e., (3) reads as "equation 3"). Ensure that any miscellaneous numbering system you use in your paper cannot be confused with a reference [4] or an equation (3) designation.

\subsubsection{Polyp}

The detection of polyps has accepted $58 \%$ by medical specialists, this can be given by the range of colors and used for this detection.

The $55 \%$ of the specialists believe that the pink (without green) has better detection, due to the color of polyp change and becomes white, yellow and green colors were selected $18 \%$ and the $9 \%$ were selected blue color by medical specialists.

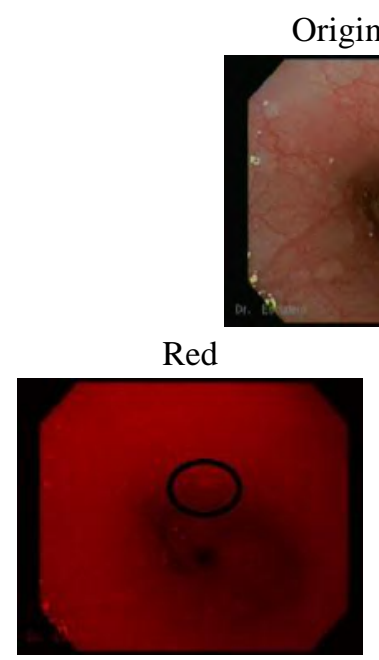

Blue
Green

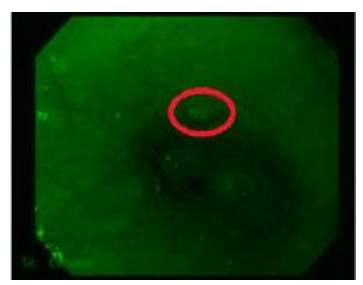

Yellow

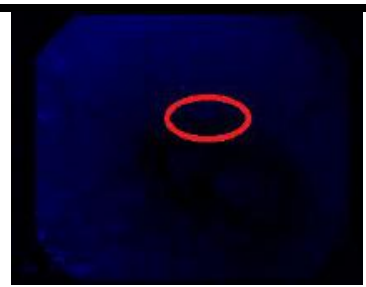

Blue Green

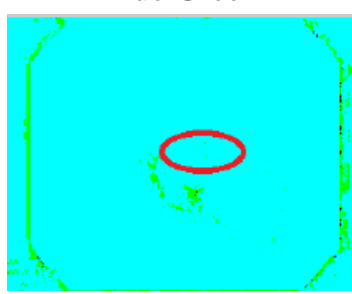

Fig.7: RGB Decomposition of Polyp

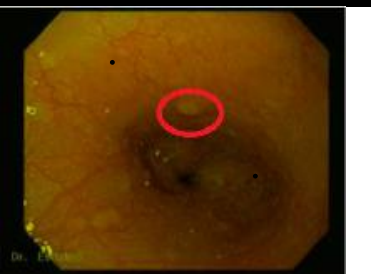

Pink

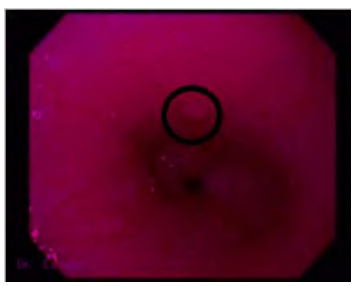

Red and green-blue (without red) colors were not succeeded in the detection of polyps, because these colors show colorimetry as walls cavity.

According to the analysis made to each injury, the following table was summarized:

Table.2: Colorimetry - Injury summary

\begin{tabular}{|l|r|r|r|r|}
\hline \multicolumn{1}{|c|}{ COLOR } & \multicolumn{1}{c|}{$\begin{array}{c}\text { Varicose } \\
\text { veins }\end{array}$} & \multicolumn{1}{c|}{$\begin{array}{c}\text { Gastric } \\
\text { ulcer }\end{array}$} & \multicolumn{1}{c|}{ Polyp } & \multicolumn{1}{c|}{ TOTAL } \\
\hline RED & $50 \%$ & $0 \%$ & $0 \%$ & $15 \%$ \\
\hline GREEN & $0 \%$ & $0 \%$ & $18 \%$ & $10 \%$ \\
\hline BLUE & $0 \%$ & $0 \%$ & $9 \%$ & $5 \%$ \\
\hline YELLOW & $17 \%$ & $67 \%$ & $18 \%$ & $25 \%$ \\
\hline PINK & $33 \%$ & $33 \%$ & $55 \%$ & $45 \%$ \\
\hline BLUE GREEN & $0 \%$ & $0 \%$ & $0 \%$ & $0 \%$ \\
\hline
\end{tabular}

The pink (without green) is the most accepted by the specialists for gastrointestinal injuries detection, due to it highlights the natural color skin, reducing interference caused by fluids on internal cavity.

The varicose veins have been denoted by the internal high turbulent flow using the red color.

\section{CONCLUSIONS}

The decomposition RGB video endoscopy allows to specialist gives faster and trusty diagnosis with the use of the specific spectra for each lesion.

Use of colors such as red and pink (without green) will allow a better detection of gastrointestinal varicose veins. Gastric ulcers are detected easily by yellow (without blue) and pink colors.

RGB decomposition is not affable to detect polyps because colorimetry doesn't show this condition.

Green-blue (without red) color is not suitable to detect gastrointestinal diseases because saturation of color given for inner fluids in the cavity.

The processing time of the system is directly linked to the characteristics of the computer, being the processor and the video card the main factors. 
The RGB decomposition of red color for the varicose veins and yellow color for ulcers can start point through systems such as: artificial intelligence, neural networks and others, to generate systems for detecting injury of this type without the intervention of medical specialist.

\section{REFERENCES}

[1] Gross, S., \& Kollenbrandt, M. (2009). Technical Evolution of Medical Endoscopy. Acta Polytechnica: Journal of Advanced Engineering, 49(2), 6-10.

[2] Reuter, M. (2006). Philipp Bozzini (1773-1809): The endoscopic idealist. Der Urologe. Ausg. A, 45(9), 1084-1088, 1090-1091. https://doi.org/10.1007/s00120-006-1165-9

[3] Doglietto, F., Prevedello, D. M., Jane, J. A., Han, J., \& Laws, E. R. (2005). Brief history of endoscopic transsphenoidal surgery--from Philipp Bozzini to the First World Congress of Endoscopic Skull Base Surgery. Neurosurgical Focus, 19(6), E3. https://doi.org/10.3171/foc.2005.19.6.4

[4] Léger, P. (2004). [Antonin Jean Desormeaux]. Progrès En Urologie: Journal de l'Association Française d'urologie et de La Société Française d'urologie, 14(6), 1231-8.

[5] Matteson, E. L. (2012). History of vasculitis: The life and work of Adolf Kussmaul. Cleveland Clinic Journal of Medicine. https://doi.org/10.3949/ccjm.79.s3.12

[6] Zajaczkowski, T. (2008). Johann Anton von MikuliczRadecki (1850-1905)-a pioneer of gastroscopy and modern surgery: His credit to urology. World Journal of Urology. https://doi.org/10.1007/s00345-0070227-y

[7] Linder, T. E., Simmen, D., \& Stool, S. E. (1997). Revolutionary Inventions in the 20th Century: The History of Endoscopy. Arch Otolaryngol Head Neck Surg, 123(11), 1161-1163. https://doi.org/10.1001/archotol.1997.019001100110 01

[8] Nabh, A., Sherid, M., \& Spurr, C. (2013). Diagnostic Endoscopy. Endoscopy of GI Tract, 37-74. https://doi.org/10.1117/1.JBO.19.4.049902

[9] Wang, A., Banerjee, S., Barth, B. A., Bhat, Y. M., Chauhan, S., Gottlieb, K. T., ... Rodriguez, S. A. (2013). Wireless capsule endoscopy. Gastrointestinal Endoscopy, 78(6), 805-815. https://doi.org/10.1016/j.gie.2013.06.026

[10] Charisis, V. S., Hadjileontiadis, L. J., Liatsos, C. N., Mavrogiannis, C. C., \& Sergiadis, G. D. (2012). Capsule endoscopy image analysis using texture information from various colour models. Computer Methods and Programs in Biomedicine, 107(1), 6174. https://doi.org/10.1016/j.cmpb.2011.10.004
[11] Oh, J., Hwang, S., Lee, J., Tavanapong, W., Wong, J., \& de Groen, P. C. (2007). Informative frame classification for endoscopy video. Medical Image Analysis, 11(2), 110-127. https://doi.org/10.1016/j.media.2006.10.003

[12]Lai, K., Bo, L., Ren, X., \& Fox, D. (2011). A largescale hierarchical multi-view RGB-D object dataset. In Proceedings - IEEE International Conference on Robotics and Automation (pp. 1817-1824). https://doi.org/10.1109/ICRA.2011.5980382

[13] Owo Eko Cahyono, Agung Tjahjo Nugroho, Miftahul Rizkiyani(2017).Detection of Paddies Reflectance Values to classify their Ages using RGB Photograph Images. International Journal of Advanced Engineering Research and Science (ISSN : 23496495(P) | 2456-1908(O)),4(10), 046-050. http://dx.doi.org/10.22161/ijaers.4.10.7

[14]Liu, Y. X., Huang, L. Y., Bian, X. P., Cui, J., Xu, N., \& Wu, C. R. (2008). Fuji Intelligent Chromo Endoscopy and staining technique for the diagnosis of colon tumor. Chinese Medical Journal, 121(11), 977982.

[15] Gono, K., Obi, T., Yamaguchi, M., Ohyama, N., Machida, H., Sano, Y., ... Endo, T. (2004). Appearance of enhanced tissue features in narrowband endoscopic imaging. Journal of Biomedical Optics, 9(3), 568. https://doi.org/10.1117/1.1695563 